\title{
RESPUESTAS INMUNOLÓGICAS, ESTABILIDAD LISOSOMAL Y FRECUENCIA DE MICRONÚCLEOS EN Eurythoe complanata (POLYCHAETA: AMPHINOMIDAE) EXPUESTOS A UNA FRACCIÓN ACUOSA DE LUBRICANTES USADOS DE MOTORES DE AUTOMÓVILES
}

\author{
Edgar ZAPATA-VÍVENES*, Leida DEL VALLE-MARCANO y Vanessa ACOSTA
}

Departamento de Biología, Escuela de Ciencias, Avenida Universidad, Cerro Colorado, Cumaná Estado Sucre, Código Postal 6101, Núcleo de Sucre, Universidad de Oriente, Venezuela

*Autor para correspondencia; ezapatavivenes@gmail.com

(Recibido febrero 2017; aceptado agosto 2017)

Palabras clave: aceites usados, celomocitos, daño celular, inmunotoxicidad, xenobióticos

\section{RESUMEN}

La viabilidad y número total de celomocitos (NTC), actividad fagocítica y de lisozimas, estabilidad de las membranas lisosomales (EML), y frecuencia de micronúcleos (FMN) en celomocitos fueron evaluados en el poliqueto Eurythoe complanata expuesto a una fracción acuosa de lubricantes usados de motores de automóviles (FALUMA) al $3 \%$ y a $200 \mu \mathrm{g} / \mathrm{L}$ de cadmio (Cd), de manera independiente, durante 21 días. Adicionalmente, se estimaron la masa corporal y los cocientes moleculares de crecimiento (proteína/ARN, proteína/ADN y ARN/ADN). El NTC y la actividad fagocítica disminuyeron en los poliquetos en presencia de FALUMA, asociados con mayor desestabilización de las membranas lisosomales y FMN, lo cual no ocurrió en los organismos expuestos a Cd. La actividad bacteriolítica de lisozimas, la masa corporal y los cocientes proteína/ARN y ARN/ADN no variaron entre los organismos experimentales; sólo se mostró un ligero incremento de proteína/ADN en organismos expuestos a FALUMA, lo que indica aumento de la síntesis de proteínas. Los parámetros celulares e inmunológicos, EML, FMN y cociente proteína/ADN en el poliqueto E. complanata presentaron sensibilidad a la mezcla de xenobióticos hidrosolubles, por lo que constituyen biomarcadores importantes en la evaluación de ambientes impactados por esta mezcla de contaminantes.

Key words: crankcase oil, coelomocytes, cellular damage, immunotoxicity, xenobiotic

\begin{abstract}
Cell viability, total coelomocytes count (TCC), phagocytosis and lysozymes activity, lysosomal membrane stability, and micronucleus frequency were determined in coelomocytes of the polychaete Eurythoe complanata exposed to $3 \%$ of water-soluble fraction of crankcase oil (WSFCO) or to $200 \mu \mathrm{g} \mathrm{Cd} / \mathrm{L}$, during 21 days. Additionally. Body mass and molecular ratios of growth (protein/RNA, protein/DNA and RNA/ DNA) were determined. Viability, TCC and phagocytosis decreased in polychaete exposed to WSFCO associated to an increased destabilization of lysosomal membranes and micronucleus frequency (MNF), but not in organisms exposed to Cd lysozyme activity. Body mass and the indices protein/RNA and RNA/DNA did not vary among
\end{abstract}


the experimental organisms; an increment of protein/DNA was only observed among organisms exposed to WSFCO, indicating an elevation of protein synthesis. The cellular and molecular responses evaluated constitute important biomarkers for the evaluation of environments impacted by these mixtures of xenobiotics.

\section{INTRODUCCIÓN}

Los lubricantes usados de motores de automóviles contienen altas concentraciones de hidrocarburos aromáticos policíclicos, compuestos clorados, metales pesados, y otras sustancias provenientes del combustible que resultan potencialmente tóxicas para la biota (Nusetti et al. 2005). Una vez que se han removido del cárter del motor, los lubricantes usados se almacenan, y en algunos casos se vierten de manera directa en los desagües de estaciones de servicios, autolavados y talleres mecánicos, teniendo a los ecosistemas marino-costeros como receptáculo final.

Los poliquetos se han usado frecuentemente como modelos biológicos en estudios ecotoxicológicos, siendo reconocidos como organismos centinelas de contaminación del bento marino (Reish y Gerlinger 1997, Dean 2008). El poliqueto Eurythoe complanata, representante de la familia Amphinomidae, ha sido uno de los más empleados en el desarrollo de protocolos experimentales para evaluaciones de efectos de sustancias xenobióticas (Rodríguez y Escaplés 1996). En esta especie se han estimado efectos de contaminantes sobre marcadores biológicos en condiciones controladas de laboratorio. Por ejemplo, la sobrevivencia y capacidad regenerativa en ejemplares expuestos a $\mathrm{Cd}$ y DDT (Reish et al. 1989), alteraciones corporales con concentraciones de Hg (Vásquez-Núñez et al. 2007), inducción de metalotioneínas en presencia de Cu y Zn (Marcano et al. 1996), estrés oxidativo en organismos contaminados con $\mathrm{Cu}$ (Nusetti et al. 2001) y aceite usado de motores de vehículos (Nusetti et al. 2005), así como respuestas moleculares e inmunológicas a exposiciones a $\mathrm{Cu}$ (Nusetti et al. 1998, Zapata-Vívenes et al. 2005).

Se ha demostrado que las funciones inmunológicas son sensibles a contaminantes químicos, por lo que su uso como biomarcadores resulta potencialmente útil en anélidos (Dhainaut y Scaps 2001). El sistema inmunológico de E. complanata consta de un componente celular (celomocitos), el cual desempeña un papel clave durante los procesos de inflamación, cicatrización de heridas y fagocitosis de patógenos, y de un componente humoral, que comprende actividad bacteriolítica mediada por lisozimas, factores aglutinantes y hemolisinas (Cuvillier-Hoet et al. 2014). Se conoce que las funciones y el balance del sistema inmunitario, tanto en invertebrados como vertebrados, pueden alterarse como resultado de exposición a contaminantes (Nusetti et al. 2004, Zapata-Vívenes et al. 2005, Marcano et al. 2006, Mydlarz et al. 2006).

Los efectos citotóxicos de xenobióticos también pueden estimarse por alteraciones de la estabilidad de membranas lisosomales, y se considera que el grado de desestabilización es proporcional a la magnitud del estrés (Lowe et al. 1995, Weeks y Svendsen 1996). El daño celular se valora mediante la retención de colorantes supravitales, presentándose una relación entre la reducción del tiempo de retención del colorante y el deterioro de las membranas lisosomales en células de organismos impactados por xenobióticos (Fang et al. 2008). Adicionalmente, la aparición de micronúcleos en celomocitos se ha recomendado como biomarcador no específico de genotoxicidad, ya que indica anormalidades nucleares o daños cromosomales acumulados durante su ciclo celular (Dixon et al. 2002).

El crecimiento evaluado a través de los cocientes moleculares proteína/ADN, proteína/ARN y ARN/ ADN permite estimar la condición fisiológica de animales en presencia de contaminantes (Acosta y Lodeiros 2003, Zapata-Vívenes et al. 2015). En esta investigación se realizó un bioensayo de exposición a largo plazo, usando una fracción acuosa de lubricantes usados de motores de automóviles (FALUMA) y cadmio para medir la competencia del sistema inmunológico innato (parámetros celulares, fagocitosis y actividad de la lisozima), la estabilidad de las membranas lisosomales y la presencia de micronúcleos en celomocitos, además del crecimiento somático en E. complanata con base en los cocientes entre proteínas, ARN y ADN.

\section{MATERIALES Y MÉTODOS}

\section{Organismos}

Se recolectaron manualmente ejemplares adultos del poliqueto E. complanata $(1.64 \pm 0.89 \mathrm{~g})$ mediante 
buceo autónomo a profundidades de 0 a $5 \mathrm{~m}$ en aguas someras de la franja costera de la zona sur del Golfo de Cariaco, en las adyacencias de la ensenada de Turpialito ( $\left.11^{\circ} 22^{\prime} 30^{\prime \prime} \mathrm{N}, 64^{\circ} 04^{\prime} 50^{\prime \prime} \mathrm{O}\right)$. Esta especie es relativamente abundante en la zona y la población de poliquetos no fue afectada por la recolección. Los individuos fueron transportados en envases de aislamiento térmico hasta el laboratorio y posteriormente se aclimataron durante dos semanas en acuarios (un individuo por cada $4 \mathrm{~L}$ de agua de mar) con arena tamizada $(50 \mathrm{~mm})$, temperatura del agua de $26 \pm 1{ }^{\circ} \mathrm{C}$ y con aireamiento continuo. Los organismos se alimentaron con tejidos congelados del cangrejo Carcinus sp. ( $200 \mathrm{mg} / \mathrm{d} / \mathrm{animal})$.

\section{Ensayo subletal}

De manera separada, se expusieron 30 ejemplares por cada tratamiento durante un periodo de 21 días a: 1) una FALUMA al $3 \% \mathrm{v} / \mathrm{v}, 2) 200 \mu \mathrm{g} / \mathrm{L}$ de $\mathrm{Cd}$ (fuente $\mathrm{CdCl}_{2} .5 \mathrm{H}_{2} 0$ ) y 3 ) sólo agua de mar en organismos testigos. El experimento se realizó por triplicado. La sobrevivencia de los organismos fue de un $100 \%$ para ambas sustancias. Los organismos se alimentaron ad libitum $2 \mathrm{~h}$ antes de la renovación del sistema (sedimento, agua y xenobióticos). El recambio se realizó cada tercer día para minimizar los efectos de productos de excreción y descomposición de alimentos.

\section{Obtención de celomocitos}

Se procedió a recolectar el fluido celómico por punción directa en el celoma usando una jeringa hipodérmica de $1 \mathrm{~mL}$ de capacidad, esterilizada, con aguja calibre 20, con un contenido de $0.5 \mathrm{~mL}$ agua de mar estéril filtrada a miliporo $(45 \mu \mathrm{m})$ y $4 \mathrm{mmol} / \mathrm{Lde}$ ácido etilendiamino-tetraacético (EDTA). El fluido extraído $(\sim 0.3 \mathrm{~mL})$ se transfirió posteriormente a tubos de microcentrífuga Eppendorf de $1.5 \mathrm{~mL}$ de capacidad, y se mantuvo a una temperatura de $10^{\circ} \mathrm{C}$. Luego se centrifugó a $1000 \mathrm{~g}$ durante $10 \mathrm{~min}$ a $4{ }^{\circ} \mathrm{C}$ y se tomó la fracción precipitada, la cual fue resuspendida en $1 \mathrm{~mL}$ de agua de mar filtrada con EDTA.

\section{Viabilidad, número total de celomocitos y fago- citosis \\ La viabilidad se determinó por tinción diferencial con colorante azul de tripano al $0.4 \%$ (Nusetti et al. 1998). Se mezclaron $10 \mu \mathrm{L}$ de la suspensión final de celomocitos con un volumen igual de azul de tripano, se resuspendió y se contaron las células en un hemocitómetro por microscopia de luz $(400 \times)$. La viabilidad se expresó como porcentaje de células}

vivas. El NTC por $\mathrm{mL}$ se determinó en los cuatro cuadrantes de la cámara y se calculó de acuerdo con la fórmula NTC $\times 10^{4} / 2$ (Goven et al. 1994a). Adicionalmente, una concentración de $1 \times 10^{6}$ por $\mathrm{mL}$ de celomocitos fue incubada con una suspensión de Saccharomyces cerevisiae a $8^{\circ} \mathrm{C}$ durante $6 \mathrm{~h}$. Se calculó el porcentaje de celomocitos que contenían al menos una levadura o resto de ellas en su citoplasma (Goven et al. 1996).

\section{Lisozima}

La actividad de la lisozima se determinó mediante el método propuesto por Goven et al. (1994b). Alícuotas de $40 \mu \mathrm{L}$ del fluido celómico se dispensaron en pocillos de $5 \mathrm{~mm}$ de diámetro en lisoplacas de agarosa al $10 \%$ en un amortiguador de fosfato $(\mathrm{pH}$ 7.5-7.8) con contenido de Micrococus lysodeikticus $(0.6 \mathrm{mg}$ células $/ \mathrm{mL})$ como substrato en placas de Petri. Las placas se incubaron a $24^{\circ} \mathrm{C}$ por $48 \mathrm{~h}$. Los diámetros de las zonas de lisis se midieron y compararon con los producidos por la lisozima de aves estándar (Sigma Chemical).

\section{Ensayo de micronúcleos}

Se realizó un fijado de células en una mezcla de metanol y ácido acético (3:1) (UNEP 1999) durante 10 a $15 \mathrm{~min}$. El frotis fue coloreado con Giemsa al 3 $\%$ durante $30 \mathrm{~min}$, y lavado posteriormente con agua bidestilada. Se dejó secar a temperatura ambiente. Las observaciones se realizaron con microscopia de luz a $1000 \times$ con aceite de inmersión. Se consideró que las células con presencia de micronúcleos (MN) eran aquellas que presentaban núcleos intactos con diámetro cercano a $1 / 3$ del diámetro en contraste con el núcleo principal. Se examinaron alrededor de 1000 células por frotis.

\section{Estabilidad de la membrana lisosomal}

Se transfirieron $40 \mu \mathrm{L}$ de líquido celómico a un portaobjetos y se mantuvieron 15 min en cámara húmeda (en oscuridad). Se colocaron $40 \mu \mathrm{L}$ de rojo neutro disuelto en dimetil sulfóxido en la lámina durante $15 \mathrm{~min}$. Las observaciones se realizaron al microscopio $(400 \times)$ cada 15 min por una hora $y$ cada 30 min en las horas subsiguientes ( $\sim 2$ horas). Se registró el tiempo máximo en que las células permanecieron sin alteración. Las observaciones no fueron mayores a 1 min para evitar el sobrecalentamiento de la muestra. Las células se examinaron con relación a las anormalidades estructurales que presentan en el tiempo de retención de rojo neutro, estimándose la proporción de células que presentan el colorante en el citosol (Lowe et al. 1992, 1995). 


\section{Cocientes de proteínas, ARN y ADN}

El tejido muscular fue homogenizado en proporción $1: 10$ en buffer salino $\left(\mathrm{NaHPO}_{4} / \mathrm{Na}_{2} \mathrm{HPO}_{4}\right)$ a 100 $\mathrm{mmol} / \mathrm{L}$. A un $\mathrm{mL}$ de extracto homogenizado se le añadió $1 \mathrm{~mL}$ de ácido perclórico (APC) al $0.4 \mathrm{~N} \mathrm{y}$ se dejó reposar por $1 \mathrm{~h}$ a $8{ }^{\circ} \mathrm{C}$. El precipitado se lavó con APC al $0.1 \mathrm{~N}$ y se disolvió con $\mathrm{KOH}$ al $0.3 \mathrm{~N}$ a $37^{\circ} \mathrm{C}$ durante $1 \mathrm{~h}$. Después de este tiempo se extrajo una alícuota para determinación de proteínas por el método de Lowry et al. (1951), usando albúmina de suero de bovino como estándar. El ARN y ADN se separaron mediante precipitación con $1 \mathrm{~mL}$ de APC 1 $\mathrm{N}$ con centrifugación a $4{ }^{\circ} \mathrm{C}$, usando el sobrenadante y el precipitado como fuente de ARN y ADN, respectivamente. El precipitado se calentó con APC a $80^{\circ} \mathrm{C}$ por un periodo de $20 \mathrm{~min}$. El ARN se determinó por el método de revelación por orcinol (Cambell y Sargent 1967), y el ADN mediante la reacción de difenilamina (Burton 1956). Se usó como sustancia referencial ARN de levadura (S. cerevicea) y ADN de timo de bovino. Adicionalmente, se estimaron los cocientes de crecimiento instantáneo proteínas/ADN, proteínas/ARN y ARN/ADN (Bulow 1987).

\section{Tasa de crecimiento}

Los animales expuestos a FALUMA, Cd y testigos fueron pesados antes y después de cada período de exposición. La tasa de crecimiento se determinó por la ecuación de Martin (1986).

\section{Análisis estadísticos \\ Para el procesamiento de datos se usó un análisis de varianza simple mediante el programa computa- rizado STATGRAPHIC PLUS versión 5.1 (ambiente Windows). Para el contraste a posteriori se aplicó una prueba de Bonferroni (Sokal y Rohlf 1979).}

\section{RESULTADOS}

La viabilidad celular no mostró diferencias significativas en los poliquetos tratados en comparación con los testigos ( $\mathrm{Fs}=1.69 ; \mathrm{p}>0.05)$. Sin embargo, el NTC presentó sus promedios más bajos en los poliquetos expuestos a FALUMA, observándose diferencias significativas en comparación con los organismos expuestos a $\mathrm{Cd}$ y los testigos ( $\mathrm{Fs}=3.94$, $\mathrm{p}<0.05$; Cuadro I). De igual manera, se encontraron diferencias significativas en la actividad fagocítica, la cual mostró un reducido número de fagocitos (1.56 veces) en los poliquetos expuestos a FALUMA ( $\mathrm{Fs}=$ $6.0, \mathrm{p}<0.05)$. Sin embargo, la actividad bacteriolítica de lisozimas no fue afectada por los tratamientos de Cd y FALUMA ( $\mathrm{Fs}=1.57, \mathrm{p}>0.05)$ (Cuadro I). Se evidenció una frecuencia de $2.4 \mathrm{MN} / 100$ células sólo en los organismos expuestos a FALUMA ( $\mathrm{Fs}=$ $14.0 ; \mathrm{p}<0.05)$. En cambio, el tratamiento con $\mathrm{Cd}$ no produjo variaciones en comparación con los organismos testigos (Cuadro I).

El mayor porcentaje de celomocitos con desestabilización en sus membranas lisosomales (DML) se observó en los poliquetos expuestos a FALUMA (Fig. 1), los cuales alcanzaron promedios mayores al $50 \%$ de daño celular en un lapso de 120-180 min. Se encontraron diferencias significativas $(\mathrm{Fs}=16.77, \mathrm{p}$ $<0.05)$ en los tiempos de retención del rojo neutro para cada tratamiento, que fueron de $328.69 \mathrm{~min}$, $191.1 \mathrm{~min}$ y $172.91 \mathrm{~min}$ para organismos testigos, expuestos a Cd y FALUMA, respectivamente.

Los niveles de proteínas expresados con base en ADN (PT/ADN) en organismos expuestos a FALUMA mostraron incremento estadístico $(\mathrm{Fs}=5.60, \mathrm{p}$ $>0.05)$, no así los cocientes proteína/ARN y ARN/ ADN (Fig. 2). El crecimiento corporal, con relación

CUADRO I. VIABILIDAD CELULAR (\%), NÚMERO TOTAL DE CELOMOCITOS $\left(\right.$ NTC $\left.\times 10^{4}\right)$, NÚMERO DE FAGOCITOS, ACTIVIDAD DE LISOZIMAS (U/mL) Y FRECUENCIA DE MICRONÚCLEOS (FMN) EN Eurythoe complanata EXPUESTOS A UNA FRACCIÓN ACUOSA DE LUBRICANTES USADOS DE MOTORES DE AUTOMÓVILES (FALUMA) DURANTE 21 DÍAS

\begin{tabular}{|c|c|c|c|}
\hline & Testigos & $\mathrm{Cd}$ & FALUMA \\
\hline Viabilidad (\%) & $94.33 \pm 1.69$ & $91.61 \pm 3.31$ & $89.05 \pm 7.77$ \\
\hline NTC & $194.5 \pm 29.28$ & $211.83 \pm 23.40$ & $172.0 \pm 32.26^{*}$ \\
\hline Fagocitos & $23.84 \pm 3.82$ & $25.83 \pm 4.02$ & $16.17 \pm 6.88^{*}$ \\
\hline Lisozima & $0.85 \pm 0.05$ & $0.86 \pm 0.03$ & $0.81 \pm 0.03$ \\
\hline FMN & $0.51 \pm 0.20$ & $1.12 \pm 0.50$ & $2.44 \pm 0.92 *$ \\
\hline
\end{tabular}

*Diferencias estadísticamente significativas al $95 \%$ 


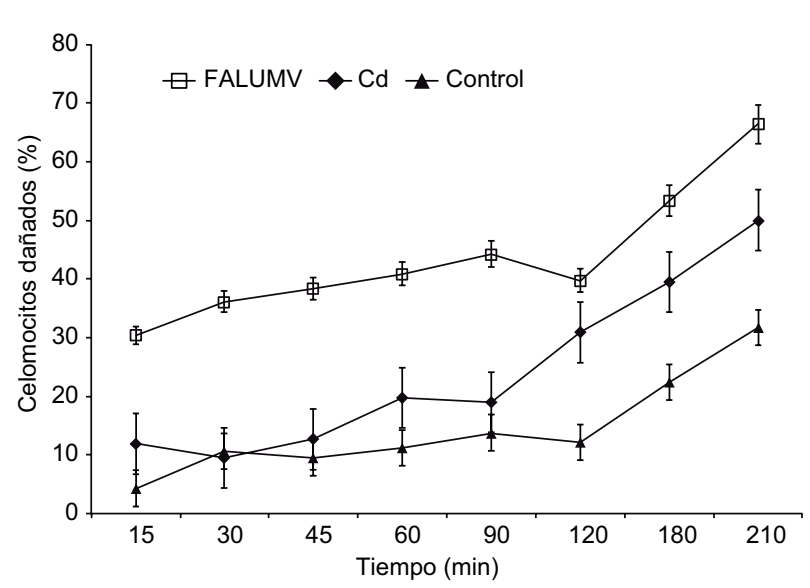

Fig. 1. Porcentaje de celomocitos con membranas lisosomales desestabilizadas en Eurythoe complanata expuestos a una fracción acuosa de lubricantes usados de motores de automóviles (cuadrados vacíos), Cd (diamantes oscuros) y testigos (triángulos oscuros) durante 21 días. La zona punteada muestra la estimación del daño al $50 \%$ de la población de celomocitos. Las extensiones denotan las desviaciones estándares

a su cambio de masa, no varió estadísticamente entre los organismos tratados ( $\mathrm{Fs}=2.04, \mathrm{p}>0.05)$, mostrando promedios de $10.32 \pm 2.34,11.2 \pm 4.30$ y 8.43 $\pm 5.49 \mathrm{~g}$ para testigos expuestos a Cd y FALUMA, respectivamente.

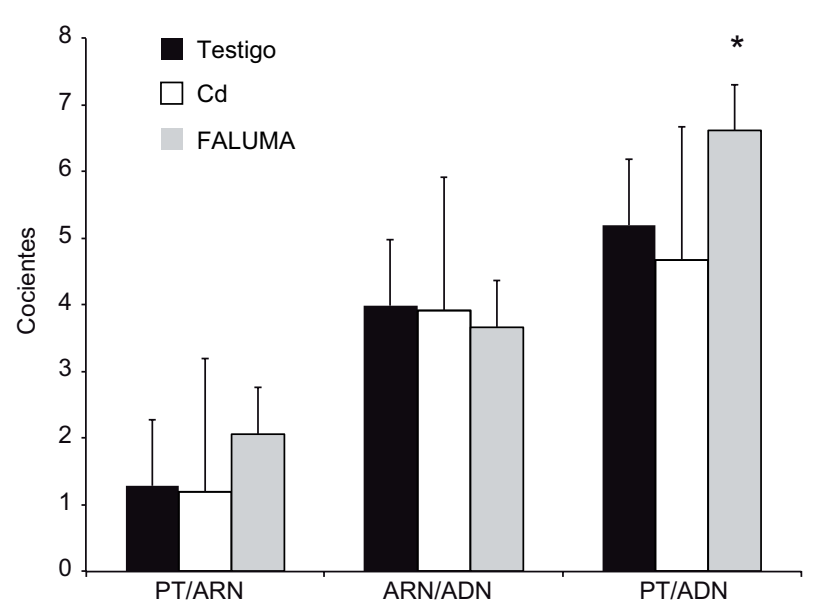

Fig. 2. Cocientes de proteína (PT) y ácidos nucleicos: PT/ ARN, ARN/ADN y PT/ADN en Eurythoe complanata expuestos a una fracción acuosa de lubricantes usados de motores de automóviles (FALUMA) y Cd por 21 días. Barras oscuras: organismos testigos; barras vacías: organismos expuestos a Cd; barras grises: organismos expuestos a FALUMA durante 21 días, con sus respectivas desviaciones estándar. Los asteriscos $(*)$ muestran diferencias estadísticamente significativas al $95 \%$

\section{DISCUSIÓN}

Los resultados revelaron que la FALUMA afectó algunas respuestas del sistema de defensa inmunológico-celular, fundamentado en la reducción del NTC y la actividad fagocítica. Adicionalmente, esta mezcla de xenobióticos hidrosolubles promovió la DML y la FMN en celomocitos. La disminución del NTC circundante refleja el efecto de los contaminantes sobre la producción celular por parte de los tejidos celomopoyéticos, lo que podría mermar la disponibilidad de fagocitos para la defensa inmunitaria, aumentando posiblemente los riesgos de infecciones e incidiendo negativamente en la condición de salud de los organismos contaminados.

La fagocitosis mostró una tendencia a disminuir en organismos contaminados con FALUMA. La supresión de la actividad fagocítica pareciera ser una respuesta común en organismos expuestos a xenobióticos orgánicos e inorgánicos. Se ha observado inmunosupresión en distintas especies en presencia de fracciones acuosas de formulaciones de petróleos (Nusetti et al. 2004, Marcano et al. 2006), cobre (Marcano et al. 1997) y herbicidas (Cortesía et al. 2015). Además, se han reportado efectos perjudiciales en distintos invertebrados marinos con exposición crónica a distintas sustancias contaminantes (hidrocarburos aromáticos, pesticidas, bifenilos policlorados, entre otros), los cuales incluyen efectos negativos sobre esta respuesta inmunitaria (Brousseau et al. 2000, Fournier et al. 2000, Sauvé et al. 2002). De igual manera, los eventos de cicatrización de heridas y regeneración tisular, respuestas corporales vinculadas íntimamente con la acción del sistema inmunológico, son alteradas en E. complanata por la presencia de metales pesados (Cd, Cu) y FALUMA (Reish et al. 1989, Nusetti et al. 2005, Zapata-Vívenes et al. 2005).

En invertebrados, el proceso fagocítico es llevado a cabo por celomocitos granulares. Tales células poseen lisosomas que pueden fundirse con las vesículas endosómicas o fagosomas, liberando una diversidad de factores inmunológicos con la finalidad de neutralizar y destruir a los agentes invasores tales como enzimas hidrolíticas, factores microbianos y especies reactivas del oxígeno (Goven et al. 1996). Este mecanismo de defensa inmunológico innato podría verse limitado por daño a las membranas lisosomales, ya sea por acción directa de los contaminantes contenidos en la FALUMA o subproductos de su metabolismo. Se conoce que la FALUMA puede inducir una condición de estrés oxidativo en E. complanata (Nusetti et al. 2005), lo que podría tener como blanco primario las membranas lisosomales de los celomocitos. 
La lisozima no presentó variaciones en su actividad en los organismos tratados, garantizando así actividad antimicrobiana. En otras investigaciones se ha demostrado que en E. complanata la actividad de la lisozima es inhibida por $\mathrm{Cu}$, inclusive se altera la capacidad de los celomocitos de formar rosetas secretorias y eritrocíticas (Marcano et al. 1997, Nusetti et al. 1998). Se han reportado observaciones similares para los anélidos terrestres Lumbricus terrestis contaminada con bifenilos policlorados (Rodríguez-Grau et al. 1989, Goven et al. 1994) y E. foetida expuesta a pesticidas organofosforados (Cortesía et al. 2015). Sin embargo, en E. complanata se han observado incrementos en la actividad de lisozima, específicamente cuando las respuestas inmunológicas-celulares se encuentran comprometidas en presencia de $\mathrm{Cu}$ (Marcano et al. 1997, Zapata-Vívenes et al. 2005). En el bivalvo $P$. imbricata, la capacidad fagocítica de los hemocitos y la actividad de la lisozima no son afectadas por contaminación subletal por una fracción acuosa de petróleo pesado (fueloil núm. 6) (Nusetti et al. 2004), aunque su actividad puede ser alterada por la concentración, el tiempo de exposición y el tipo de xenobiótico involucrado.

El sistema de membranas lisosomales en los poliquetos expuestos a FALUMA constituye un blanco subcelular susceptible, en comparación con la dosis usada de $\mathrm{Cd}$. En numerosas investigaciones se ha estimado que el tiempo de retención de rojo neutro (TRRN) en células sanguíneas en invertebrados marinos contaminados varía de 60 a $120 \mathrm{~min}$ para alcanzar el $50 \%$ de las células dañadas por el colorante (Nascimento et al. 2002). En anélidos terrestres se ha usado esta técnica para evaluar efectos de clorpirifos y glifosatos en celomocitos de E. fetida andrei, registrándose valores reducidos en los organismos expuestos a ambos agrotóxicos (Ringwood et al. 1998, Casabé et al. 2007, Piola et al. 2013). El TRRN en el camarón Lytopeneaus vannamei puede verse afectado por concentraciones relativamente bajas de $\mathrm{Cd}$, manifestando una relación dosis-respuesta (Chang et al. 2009). La presencia de metales pesados en el mejillón $P$. viridis también puede afectar este parámetro (Nicholson y Lam 2005, Zhao et al. 2011, Zapata-Vívenes et al. 2012).

La aparición de micronúcleos en los organismos expuestos a FALUMA y $\mathrm{Cd}$ es indicio de posibles aberraciones cromosómicas que pueden ocurrir (aunque en baja frecuencia) en los procesos de división celular en los celomocitos, durante las fases de premaduración en los tejidos hematopoyéticos. Este detalle revela el daño genotóxico que puede estimular la mezcla de contaminantes hidrosolubles contenidos en la FALUMA. En otros invertebrados se ha demostrado el efecto que pueden tener fracciones de hidrocarburos en la formación de micronúcleos, por lo que estos organismos son muy eficaces para detectar daños por contaminación en estudios de monitoreo costeros (Fernández et al. 2011).

El incremento en los promedios de la relación PT/ADN sugiere la activación de la síntesis de proteínas en presencia de contaminantes (ZapataVívenes et al. 2015). El aumento de la concentración de proteínas puede indicar ajustes moleculares vinculados con la formación de tales macromoléculas para la captación, defensa y desintoxicación contra compuestos metálicos y orgánicos que ingresan en la pared corporal del poliqueto; dentro de éstas se incluyen las metalotioneínas. Al igual que el crecimiento corporal de los poliquetos expuestos a $\mathrm{Cd}$ y FALUMA, los cocientes ARN/ADN y proteína/ ARN no presentaron cambios, considerando que la condición fisiológica celular no fue afectada por los tratamientos. En otros trabajos no se han encontrado cambios significativos en tales índices de crecimiento en E. complanata (Nusetti et al. 1998), aunque en bivalvos se ha demostrado que tales cocientes se alterados por exposición a metales pesados (Acosta y Lodeiros 2003) y FALUMA (Zapata-Vívenes et al. 2015).

\section{CONCLUSIONES}

Se demostró que la fagocitosis, FMN y DML, así como el cociente PT/ADN, son señales sensibles en poliquetos expuestos a FALUMA, no así con relación a la dosis de $\mathrm{Cd}$. El uso de respuestas inmunológicas, genotoxicidad y cocientes moleculares de crecimiento podrían permitir la aplicación de multibiomarcadores en monitoreos ambientales de áreas impactadas por contaminantes.

\section{AGRADECIMIENTOS}

Los autores agradecen al Consejo de Investigación de la Universidad de Oriente, a la Academia de Ciencias Físicas, Matemáticas y Naturales, y al Instituto Oceanográfico de Venezuela.

\section{REFERENCIAS}

Acosta V. y Lodeiros C. (2003). Índice ARN/ADN en poblaciones de la almeja Tivela mactroides (Bivalvia: 
Veneridae) provenientes de localidades con diferentes niveles de contaminación. Revista Científica FCVLUZ. 13 (5), 378-382.

Brousseau P., Pellerin J., Morin Y., Cyr D., Blakey B., Boermans H. y Fournier M. (2000). Flow cytometry as a tool to demonstrate the disturbance of phagocytosis in the clam Mya arenaria following in vitro exposure to heavy metals. Toxicology 142 (2), 145-156.

DOI: $10.1016 / \mathrm{S} 0300-483 \mathrm{X}(99) 00165-1$

Bulow F. (1987). RNA-DNA ratios as indicator of growth in fish. En: Age and growth in fish (J.R. Summerfelt y C.S. Cobb, Eds.). Iowa State University Press, Ames, EUA. pp. 255-367.

Burton K. (1956). A study of the conditions and mechanisms of the diphenilamine reaction for the colorimetric estimation of deoxyribonucleic acid. Biochem. J. 62 (2), 315-323.

DOI: $10.1042 / \mathrm{bj} 0620315$

Cambell P.N. y Sargent J.R. (1967). Estimation of ARN by orcinol method of mejbum (1939). En: Techniques in protein biosynthesis (P.N. Cambell y J.R. Sargent, Eds.). Academic Press. Londres, Reino Unido, pp. 301-302.

Casabé N., Piola L., Fuchs J., Oneto M., Pamparato L., Basack S., Giménez R., Massaro R., Papa J. y Kesten E. (2007). Ecotoxicological assessment of the effects of glyphosate and chlorpyrifos in an Argentine soya field. J. Soils. Sedim. 7 (4), 232-239.

DOI: $10.1065 /$ jss2007.04.224

Chang M., Wang W.N., Wang A.L., Tian T.T., Wang P., Zheng Y. y Liu Y. (2009). Effects of cadmium of respiratory burst, intracellular $\mathrm{Ca}^{+2}$ and DNA damage in the white shrimp Litopenaeus vannamei. Comp. Biochem. Physiol. 149 (4), 581-586.

DOI: $10.1016 /$ j.cbpc.2008.12.011

Cortesía C., Marcano L., Marcano E. y Zapata-Vívenes E. (2015). Inmunotoxicidad de malatión y clorpirifos en la lombriz de tierra Eisenia sp. (Annelida: Oligochaeta). Saber 27 (4), 530-536.

Cuvillier-Hoet V., Boidin-Wichlacz C. y Tasiemski A. (2014). Polychaetes as annelid models to study ecoimmunology of marine organisms. J. Mar. Sci. Technol. 22 (1), 9-14. DOI: 10.6119/JMST-013-0718-1

Dhainaut A. y Scaps P. (2001). Immune defense and biological responses induced by toxics in Annelida. Can. J. Zool. 79 (1), 233-253. DOI: 10.1139/cjz-79-2-233

Dean H.K. (2008). The use of polychaetes (Annelids) as indicator species of marine pollution: A review. Rev. Biol. Trop. 56 (4), 11-38.

Dixon D.R., Pruski A.M., Dixon L.R.J. y Awadhesh N.J. (2002). Marine invertebrate eco-genotoxicology: A methodological overview. Mutagenesis 17 (6), 495-507. DOI: 10.1093/mutage/17.6.495
Fang J., Wu R., Zheng G., Lam P. y Shin P. (2008). Induction, adaptation and recovery of lysosomal integrity in green-lipped mussel Perna viridis. Mar. Pollut. Bull. 57 (6-12), 467-72.

DOI: 10.1016/j.marpolbul.2008.03.038

Fernández B., Campillo J.A., Martínez-Gómez C. y Benedicto J. (2011). Micronuclei and other nuclear abnormalities in mussels (Mytilus galloprovincialis) as biomarkers of cyto-genotoxic pollution in Mediterranean waters. Environ. Mol. Mutagen. 52 (6), 479-491. DOI: 10.1002/em.2064

Fournier M., Cyr D., Blakey B., Boermans H. y Brousseau P. (2000). Phagocytosis as a biomarker of immunotoxicity in wildlife species exposed to environmental xenobiotics. Am. Zool. 40 (3), 412-420. DOI: 10.1668/0003-1569(2000)040[0412:PAABOI ]2.0.CO;2

Goven A. J., Fitzpatrick L. y Venables B. (1994a). Chemical toxicity and host defense in earthworms. Ann. N. Y. Acad. Sci. 712 (1), 280-299. DOI: 10.1111/j.1749-6632.1994.tb33579.x

Goven A., Chen S., Fitzpatrick L. y Venables B. (1994b). Lysozyme activity in earthworm (Lumbricus terrestris) coelomic and coelomocytes: Enzyme assay for immunotoxicity of xenobiotics. Envirom. Toxicol. Chem. 3, 607-613. DOI: 10.1002/etc.5620130409

Goven A.J., Kennedy J., Gilles R. y Cooper E.L. (1996). Environmental pollution and toxicity in invertebrates: An earthworm model for inmunotoxicology. Adv. Comp. Environ. Physiol. 24, 170-211. DOI: 10.1007/978-3-642-79847-4_7

Lowe D., Moore M. y Evans B. (1992). Contaminant impact of interactions of molecular probes with lysosomes in living hepatocytes from dab Limanda limanda. Mar. Ecol. Prog. Ser. 91 (1), 135-140. DOI: 10.3354/meps091135

Lowe D., Fossato V. y Depledge M. (1995). Contaminantinduced lysosomal membrane damage in blood cells of mussel Mytilus galloprovincialis from the Venice lagoon: An in vitro study. Mar. Ecol. Prog. Ser. 129 (1), 189-196.

DOI: $10.3354 /$ meps 129189

Lowry O.H., Rosebrough N.J., Farr A.L. y Randal R.J. (1951). Protein measurement with folin phenol reagent. J. Biol. Chem. 193 (1), 265-275.

Marcano L., Nusseti O., Rodríguez Grau J. y Vilas J. (1996). Uptake and depuration of copper and zinc in relation to metal binding protein in the polychaeta Eurythoe complanata. Comp. Biochem. Physiol. 114 (3), 179-184. DOI: 10.1016/0742-8413(96)00016-3

Marcano L., Nusetti O., Rodríguez-Grau J., Briceño J. y Vilas J. (1997). Coelomic fluid lysozyme activity induction in the polychaete Eurythoe complanata as 
a biomarker of heavy metal toxicity. Bull. Environ. Contam. Toxicol. 59 (1), 22-28.

DOI: $10.1007 / \mathrm{s} 001289900438$

Marcano L., Nusetti O., Zapata-Vívenes E., Nusetti S. y Esclapés M.M. (2006). No. 6 fuel oil effects on antioxidant enzymes and immunological responses in the fish Thalassophryne maculosa (Pisces: Batrochoididae). J. Braz. Soc. Ecotoxicol. 1 (1), 31-35.

DOI: $10.5132 /$ jbse.2006.01.007

Martin N.A. (1986). Toxicity of pesticides to Allobophora caliginosa (Oligochaeta: Lumbricidae). New Zeal. J. Agr. Res. 29 (1), 699-706.

DOI: $10.1080 / 00288233.1986 .10430466$

McHenery J., Birbeck T. y Allen J. (1979). The occurrence of lysozyme in marine bivalves. Comp. Biochem. Physiol. B. 63, 25-28.

Mydlarz L.D., Jones L.E. y Drew-Harvell C. (2006). Innate immunity, environmental drivers, and disease ecology of marine and freshwater invertebrates. Annu. Rev. Ecol. Evol. Syst. 37, 251-88.

DOI: 10.1146/annurev.ecolsys.37.091305.110103

Nascimento I.A., Leite M.B y Martins L.K. (2002). Uso de biomarcadores para diagnóstico ambiental: Proteinas de estresse e interidade da membrana lisossômica. En: Métodos em ecotoxicologia marinha: Aplicações no Brasil. (I.A. Nascimento, P.M. Sousa y M. Nipper, Eds.). Artes Gráficas e Industrias, San Pablo, Brasil, 207-232.

Nicholson S. y Lam P. (2005). Pollution monitoring in Southeast Asia using biomarkers in the mytilid mussel Perna viridis (Mytilidae: Bivalvia). Environ. Inter. 31 (1), 121-132. DOI: 10.1016/j.envint.2004.05.00

Nusetti O.A., Salazar-Lugo R., Rodríguez-Grau J. y Vilas J. (1998). Immune and biochemical responses of the polychaete Eurythoe complanata exposed to sublethal concentrations of copper. Comp. Biochem. Physiol. 119C (2), 177-183.

DOI: 10.1016/S0742-8413(97)00205-3

Nusetti O., Escalpés M., Salazar G., Nusetti S. y Pulido S. (2001). Biomarkers of oxidative stress in the polychaete Eurythoe complanata (Amphinomidae) under short term copper exposure. Bull. Environ. Contam. Toxicol. 66 (5), 576-581. DOI: 10.1007/s001280047

Nusetti O., Marcano L., Zapata E., Esclapés M., Nusetti S. y Lodeiros C. (2004). Respuestas inmunológicas y de enzimas antioxidantes en la ostra perla Pinctada imbricata (Mollusca: Pteridae) expuesta a niveles subletales de fuel oil ${ }^{\circ}$ 6. Interciencia 29 (6), 324-329.

Nusetti O., Zapata-Vívenes E., Esclapés M. y Rojas M. (2005). Antioxidant enzymes and tissue regeneration in Eurythoe complanata (Polychaeta: Amphinomidae) exposed to used vehicle crankcase oil. Arch. Environ. Contam. Toxicol. 48 (4), 1-6.

DOI: $10.1007 / \mathrm{s} 00244-004-0041-0$
Piola L., Fuchs J., Oneto M.L., Basack S., Kesten E. y Casabé N. (2013). Comparative toxicity of two glyphosate-based formulations to Eisenia andrei under laboratory conditions. Chemosphere 91 (4), 545-551. DOI: 10.1016/j.chemosphere.2012.12.036

Reish D.J., Asato S.L. y Lemany J.A. (1989). The effects of cadmium and DDT on the survival and regeneration in the amphinomidae polychaete Eurythoe complanata. Proceedings of the 7th Symposium on Marine Biology, California State University, Long Beach, California, 6-11 agosto, 107.

Reish D. y Gerlinger T. (1997). A review of the toxicological studies with polychaetous annelids. Bull. Mar. Sci. 60 (2), 584-607.

Ringwood A.H., Conners D.E. y Hoguet J. (1998). Effects of natural and anthropogenic stressors on lysosomal destabilization in oyster Crassostrea virginica. Mar. Ecol. Progr. Ser. 166, 163-171.

DOI: $10.3354 /$ meps 166163

Rodríguez-Grau J., Venables B., Fitzpatric L., Goven A. y Cooper E. (1989). Suppression of secretory rosette formation PCBs in Lumbricus terrestris, and earthworm immune assays for humoral immunotoxicity of xenobiotics. Environ. Toxicol. Chem. 8 (12), 12011207. DOI: $10.1002 /$ etc. 5620081213

Rodríguez J. y Escaplés M. (1996). Protocolos estándares para bioensayos de toxicidad con especies acuáticas. Gerencia General de Tecnología del Departamento de Ecología y Ambiente, Instituto de Tecnología Venezolana para el Petróleo y Petróleo de Venezuela, Caracas, Venezuela, $109 \mathrm{pp}$.

Sauvé S., Hendawi M., Brousseau P. y Fournier M. (2002). Phagocytic response of terrestial and aquatic invertebrates following in vitro exposure to trace elements. Ecotoxicol. Environ. Saf. 52 (1), 21-29.

DOI: $10.1006 /$ eesa.2001.2125

Sokal R.R. y Rohlf F.J. (1979). Biometría: principios y métodos estadísticos en la investigación biológica. $\mathrm{H}$. Blumme, Madrid, España, 832 pp.

UNEP (1999). Manual on the biomarker recommended for the MED POL biomonitoring programme. United Nations Environment Programme. Mediterranean Action Plan (Remoge report), Atenas, Grecia, 92 pp.

Vásquez-Núñez R., Méndez N. y Green-Ruiz, C. (2007). Bioaccumulation and elimination of $\mathrm{Hg}$ in the fireworm Eurythoe complanata (Annelida:Polychaeta) from Mazatlán, Mexico. Arch. Environ. Contam. Toxicol. 52 (4), 541-548. 10.1007/s00244-006-0016-4

Weeks J. y Svendsen C. (1996). Neutral red retention by lysosomes from earthworm (Lumbricus rubellus) coelomocytes: A simple biomarker of exposure to soil copper. Environ. Toxicol. Chem. 15 (10), 1801-1805. DOI: $10.1002 /$ etc.5620151022 
Zapata-Vívenes E., Nusetti O., Marcano L., Escaplés M. y Arredondo L. (2005). Respuestas inmunológicas y cicatrización en el poliqueto Eurythoe complanata (Annelida: Amphinomidae) expuesto a cobre. Ciencias Marinas 31 (1), 1-9. DOI: 10.7773/cm.v31i11.83

Zapata-Vívenes E., Rojas-Astudillo L., Sánchez G. y Barreto M. (2012). Metales pesados y biomarcadores relacionados en Perna viridis (Bivalvia: Mytilidae) colectado en costas del estado Sucre, Venezuela. Ciencias Marinas 38 (3), 517-528.

DOI: $10.7773 / \mathrm{cm} . v 38 \mathrm{i} 3.2046$.
Zapata-Vívenes E., Sánchez G. y Marcano L. (2015). Reservas energéticas e índices moleculares de condición en Lima (Ctenoides) scabra expuesta a lubricantes usados de motores de automóviles. Zootecnia Trop. 33 (1), 37-45.

Zhao C., Xiaoxu L., Shibin L. y Chang Y. (2011). Assessments of lysosomal membrane responses to stresses with neutral red retention assay and its potential application in the improvement of bivalve aquaculture. African J. Biotechnol. 10 (64), 13968-13973.

DOI: $10.5897 / \mathrm{AJB} 10.2283$ 